Proceedings

\title{
Investigation of Application-Specific Thin Film Sensor Systems with Wireless Data Transmission System ${ }^{+}$
}

\author{
Eike Meyer-Kornblum *, Saskia Biehl, Nancy Paetsch and Günter Bräuer \\ Fraunhofer Institute for Surface Engineering and Thin Films, Bienroder Weg 54E, 38108 Braunschweig, \\ Germany; saskia.biehl@ist.fraunhofer.de (S.B.); nancy.paetsch@ist.fraunhofer.de (N.P.); \\ guenter.braeuer@ist.fraunhofer.de (G.B.) \\ * Correspondence: eike.meyer-kornblum@ist.fraunhofer.de; Tel.: +49-531-2155-764 \\ + Presented at the Eurosensors 2018 Conference, Graz, Austria, 9-12 September 2018.
}

Published: 12 December 2018

\begin{abstract}
Thin-film-based smart telemetric sensors coated onto the surface of washers were developed for the measurement of normal forces in screw connections for industrial applications.
\end{abstract}

Keywords: smart telemetric sensor; screw connections; thin film sensor, piezo-resistive, thermoresistive, screw force, wear resistance, thin film washer, sensory washer

\section{Introduction}

The fabrication of individually designed sensor systems for the static measurement of load distributions is a very important theme in many applications like wind mills, production machines, bridges or test arrangements [1,2]. For a flexible design of production equipment and testing systems the development of mobile energy self-sufficient, battery-driven and wireless sensors is of great importance. Innovative sensor modules in the shape of a washer as a long-term stable, wireless monitoring system for screw joints that can easily be integrated into existing systems meet the needs of the industry. In many screw connection applications, the sensor system shall be in the same size as the standard integrated washer. Therefore, a thin film layer system is directly coated onto a hardened steel washer-like base body. The stiffness of the system stays almost the same, because no structural changes except for the added thin film system have to bedone.

For monitoring, the resistances of different piezo- und thermo-resistive sensors, as part of the multifunctional thin film system, are determined. Inside a measuring circuit both signals are processed and send via a Bluetooth Low Energy data transmission module to a receiver.

\section{Materials and Methods}

For the metrological acquisition of temperatures and normal forces within the layer system at discrete points on the washer surface, different physical effects are used. By the piezo-resistive effect of amorphous hydrogenated carbon $(\mathrm{a}-\mathrm{C}: \mathrm{H})$ films the force on discrete points could be determined. Therefore, the a-C:H thin film, optimized for force measurement, is homogenously applied directly onto the surface of a polished washer-based work piece which is shown as black film in the schematic representation of the layer system in Figure 1. This sensory layer, called DiaForce ${ }^{\circledR}$, is deposited within a plasma enhanced chemical vapour deposition (PECVD) and achieves an average thickness of $6 \mu \mathrm{m}$. It has a high hardness of approximately $24 \mathrm{GPa}$ with a modulus of elasticity of $264 \mathrm{GPa}$, so that the deformation of the sensor layer can be neglected as well as the additional elasticity [3]. 


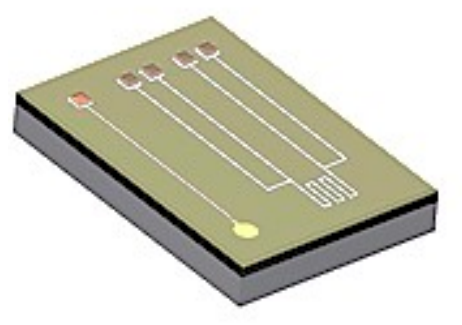

Si and $\mathrm{O}$ modified carbon layer as top layer $d=3 \mu \mathrm{m}$ and

$\square$ Cu contacts $\mathrm{d}=1,5 \mu \mathrm{m}$

$\square$ Cr-meander $\mathrm{d}=0.2 \mu \mathrm{m}$

$\square$ intermediate layer $\mathrm{d}=1 \mathrm{\mu m}$

$\square$ electrode structure $d=0,2 \mu \mathrm{m}$

a piezo-resistive hydrogenated carbon layer $\mathrm{d}=6 \mathrm{\mu m}$

$\square$ substrate

Figure 1. Schematic representation of the layer system.

On top of this piezo-resistive layer circular electrodes out of chromium are fabricated by a combination of a physical vapour deposition (PVD) process with photolithography and chemical wet etching. The thickness of the chromium layer is about $200 \mathrm{~nm}$ and shown in Figure 1 on the left hand side. The circular structures are in direct contact with the DiaForce ${ }^{\circledR}$-layer, so that the load-dependent electrical resistance of the sensor layer is measured between these electrodes and the conductive basebody. These are the areas for load measurement. For insulating the sensory layer against following measuring elements and conductor tracks, an intermediate layer of silicon- and oxygen-modified hydrocarbon (a-C:H:Si:O) is deposited in a structured manner with a layer thickness of approx. $1 \mu \mathrm{m}$ in another PECVD process on top. In a second PVD coating, the conductive tracks are deposited and structured the same way as the electrodes before. Additionally, PTC thermistors, based on the thermally induced resistance change of metallic chromium, are integrated in this structured layer (Figure 1, right hand side). These thermistors are in the geometry of temperature sensitive meandered tracks equipped with two supply and two measuring conductive tracks for four-wire measurement. The electrical contacting of all the sensor structures is accomplished by contact pads, which are placed outside the load area and provided with a solderable copper coating. Within a final PECVD process the insulation and wear protection a-C:H:Si:O layer is deposited homogenously on top with a thickness of about $3 \mu \mathrm{m}$.

\section{Results}

The precise measurement of temperature has a high importance, especially in the case of temperature change during a load measurement. The a-C:H-layer shows, as a semiconductor, a temperature sensitivity which is why the temperature compensation is very important for a precise load measurement $[3,4]$. Therefore, a direct electronic temperature compensation is implemented. Two a-C:H sensor structures, one unloaded as a temperature compensating resistor and the other within the load area as a force sensing element are interconnected as voltage dividers. Assuming that both sensors have the same thermal sensitivity and are subjected to the same temperature, the temperature influences cancel each other out. To determine these behaviour, a pre-characterisation of all sensor structures has to be done. The thermo-resistive characteristics of two force sensor structures, as well as the compensated result and a temperature meander are presented in Figure 2a. In Figure $2 \mathrm{~b}$ two different layouts are shown in comparison. Due to the semi-conductive behaviour the two a-C:H force sensors show an exponential resistance dependency on temperature. This can be described by the B parameter equation as usual with semiconductors [5]. The thermo-resistive meander structure likewise shows a simplified linear resistance dependency known of metallic resistors. Via the outer supply conductive tracks a constant current of $1 \mathrm{~mA}$ is applied and the voltage change of the meandered resistor is measured via the inner measuring conductors. 


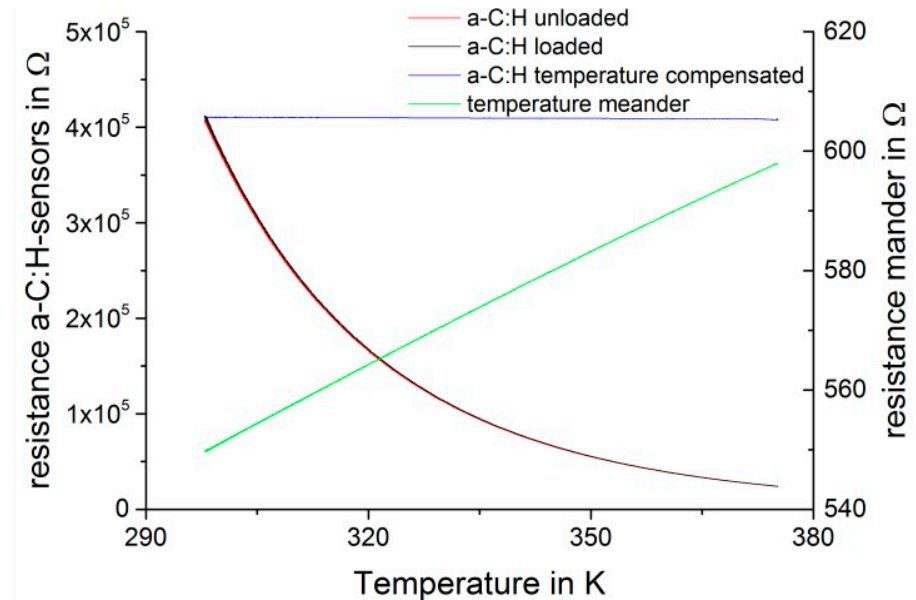

(a)

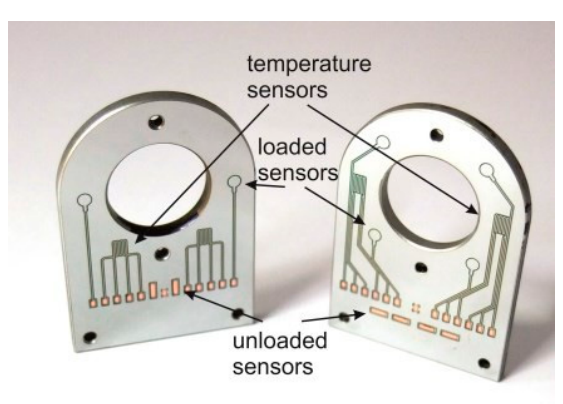

(b)

Figure 2. (a) Thermo-resistive characteristic curves of two force sensor structures, the compensated result and a temperature meander; (b) two different sensor layouts.

Different sensor layouts with a varying number of sensor structures for force and temperature measurement were investigated. For the wireless data transmission of the measured values by Bluetooth Low Energy several circuit boards are developed (Figure 3a). It consists of an analogue matching circuit with amplification, filtering and digitizing as well as a digital processing and data transmission unit, which sends the corresponding data to a receiver. That can be a mobile phone, a tablet or a personal computer. The circuit is powered by a $240 \mathrm{~mA}$ accumulator, which provides a measurement time of about $24 \mathrm{~h}$ at $20 \mathrm{~Hz}$ data rate on four force and two temperature sensors. In Figure $3 \mathrm{~b}$ the complete telemetric smart sensor system is pictured.

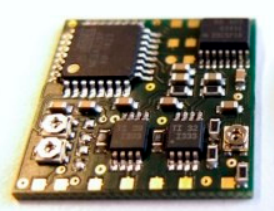

(a)

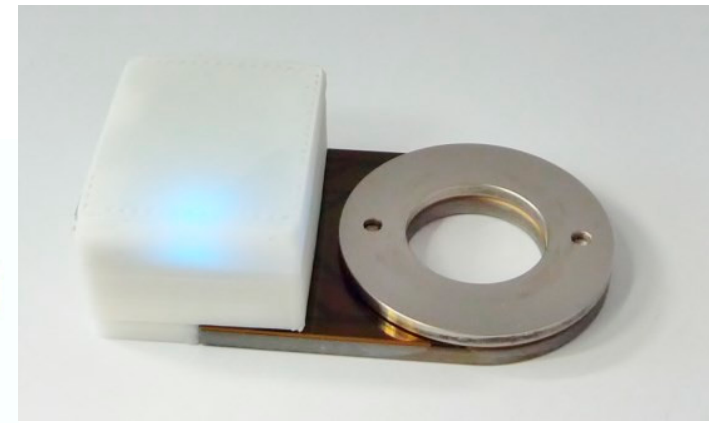

(b)

Figure 3. (a) Measuring circuit with amplification, filtering and digitizing as well as a digital processing and data transmission via Bluetooth Low Energy; (b) washer sensor with electrical measuring and data transmission circuit inside the attached housing.

The piezo-resistive characterisation shown in Figure 4 is done after the merge of the sensory washer and the measurement. It shows the load dependent characteristic of the adjusted measuring voltage of the circuit. Therefore, the sensor was loaded under a test arrangement and the corresponding sensor data were recorded with a tablet as receiving unit. In parallel the applied load was measured by a computer. Through synchronization, both series of measurements were then combined so that the correlation between the reference force and the measured voltage can be determined. 


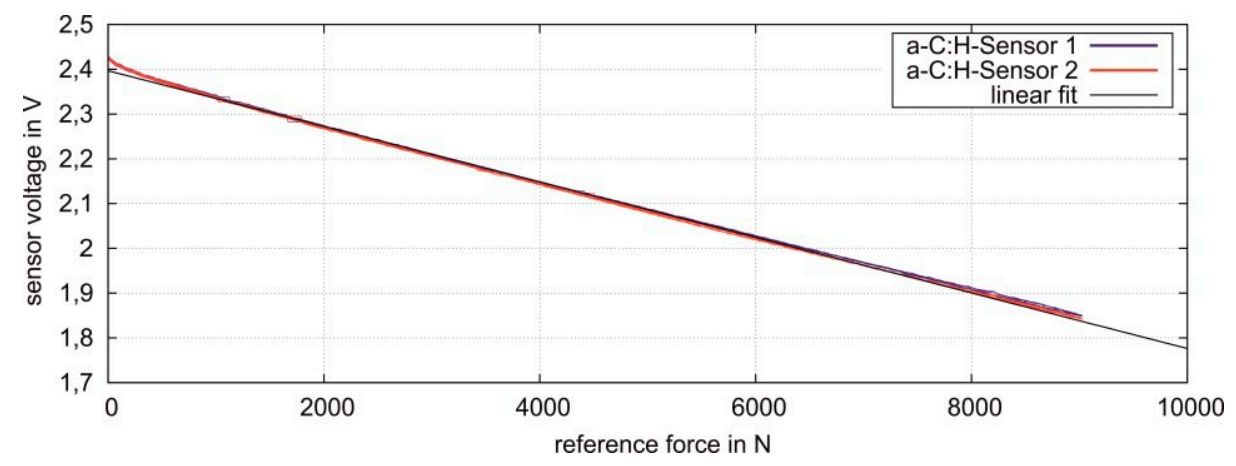

Figure 4. Load dependent characteristic of the measuring voltage transmitter by Bluetooth Low Energy.

\section{Discussion}

The results of the thermo-resistive characterisation show a major temperature influence on both, the metallic temperature sensor and the a-C:H semi-conducting thin film. With the aid of direct electronic temperature compensation, the thermal impact on the force-measuring sensors can be efficiently reduced (Figure 2a). The measurement results of the load dependent characterisation show a linear characteristic, which can easily be converted into the corresponding force value via the inverse function of the linear fit.

\section{Conclusions}

The new, long-term stable measuring and safety system uses a Bluetooth Low Energy system for data transfer. It can be integrated and adapted easily in a variety of applications. In wind power plants, as building monitoring system and production plants, it statically and dynamically records the local load distribution on top of a washer due to an adaptable number of sensor structures. In addition, the chrome meandering structure provides optional temperature sensing inside the thin film.

Author Contributions: S.B. coordinated the project, N.P. structured and fabricated all sensor layouts, E.M.-K. designed the sensor layouts, the electrical circuits and wrote the paper. G.B. is the head of the Fraunhofer IST.

Acknowledgments: The author thanks Dennie Herrmann and Jonas Stübing for the deposition of thin films and Elke Bürger for the always reliable analysis.

Conflicts of Interest: The authors declare no conflict of interest.

\section{References}

1. Biehl, S.; Rumposch, C.; Paetsch, N.; Bräuer, G.; Weise, D.; Scholz, P.; Landgrebe, D. Multifunctional thin film sensor system as monitoring system in production. Microsyst. Technol. 2016, 22, 1757-1765.

2. Meškinis, Š.; Gudaitis, R.; Vasiliauskas, A.; Čiegis, A.; Šlapikas, K.; Tamulevičius, T.; Andrulevičius, M.; Tamulevičius, S. Piezoresistive properties of diamond like carbon films containing copper. Diamond Relat. Mater. 2015, 60, 20-25.

3. Biehl, S. Entwicklung von Dünnschichtsensoren auf Basis piezoresistiver Kohlenwasserstoffschichten. Dissertation, Fraunhofer IST, Braunschweig, Germany, 2011; ISBN 978-3-8396-0354-3.

4. Hesse, S.; Schnell, G. Sensoren für die Prozess- und Fabrikautomation; Springer Fachmedien: Wiesbaden, Germany, 2014; ISBN 978-3-658-05866-1.

5. Robertson, J. Improving the properties of diamond-like carbon. Diamond Relat. Mater. 2003, 12, 79-84.

(C) 2018 by the authors. Licensee MDPI, Basel, Switzerland. This article is an open access article distributed under the terms and conditions of the Creative Commons Attribution (CC BY) license (http://creativecommons.org/licenses/by/4.0/). 